\title{
A Novel Approach to the Removal of a Retained Guidewire
}

\author{
Michael Tucciarone ${ }^{\mathrm{a}, \mathrm{b}}$, Hussein Othman ${ }^{\mathrm{a}}$, Melissa Frederick ${ }^{\mathrm{a}}$, Andrew Boguszewski ${ }^{\mathrm{a}}$, \\ Kumara Rama ${ }^{\mathrm{a}}$
}

\begin{abstract}
Retained intravascular foreign body is a rare, but well-documented complication of percutaneous procedures. We present a case of a novel approach of endovascular retrieval utilizing laparoscopic tools of a retained guidewire. A 74-year-old male presents with an implantable cardioverter defibrillator shock secondary to a retained J-wire from a prior right heart catheterization. Attempts at percutaneous retrieval were unsuccessful. A transjugular approach with laparoscopic tools was used for successful removal of the J-wire. Percutaneous intervention has made the retrieval of retained foreign bodies feasible, safe and effective in the majority of situations. However, creative endovascular approaches may be required as described in this case.
\end{abstract}

Keywords: J-Wire; Implantable Cardioverter-defibrillator (ICD); Superior Vena Cava (SVC); Inferior Vena Cava (IVC); Ventricular Tachycardia; intravascular foreign body; Autosuture Versa step $5 \mathrm{~mm}$ port; alligator cystoscope forcep

\section{Introduction}

Retained intravascular foreign body is a well-documented complication of endovascular procedures. Percutaneous approach is currently the method of choice for removal of retained objects. We report a case of transjugular extraction of an old endothelialized retained J-wire due to wire induced

Manuscript accepted for publication June 2, 2011

${ }^{\text {a }}$ St. John Hospital and Medical Center, 22101 Moross road, VEP 2nd floor cathlab, Detroit MI 48326, USA

${ }^{\mathrm{b}}$ Corresponding author: Michael Tucciarone,

Email: mtucciaron@hotmail.com

doi: $10.4021 / \mathrm{jmc} 233 \mathrm{w}$ symptomatic ventricular tachycardia with the aid of an Autosuture Versa Step $5 \mathrm{~mm}$ port.

\section{Case Report}

A 74 years old male with ischemic cardiomyopathy and ejection fraction of $20 \%$ with an intracardiac defibrillator presented with a defibrillator discharge. He was asymptomatic just prior to his device going off. There were no described palpitations, chest pain or shortness of breath. AICD interrogation showed a successfully aborted ventricular tachycardia. Physical examination revealed good overall general heath. Chest auscultation was unremarkable. Heart sounds were normal. Vascular exam showed good peripheral pulses and no edema.

To rule out coronary ischemia, cardiac catheterization was performed, which revealed stable coronary artery disease. Under fluoroscopy, a J-wire was noted to run from the superior vena cava (SVC) to the right atrium, looped through the right ventricle and into the inferior vena cava (IVC) (Fig. 1). The J-wire was also seen on a prior heart catheterization in 2007, but at that time was seen spanning from the SVC

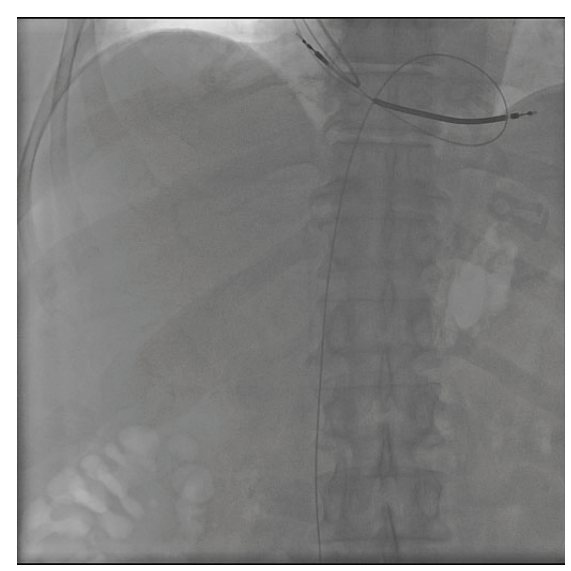

Figure 1. J-wire was noted to run from the superior vena cava (SVC) to the right atrium, looped through the right ventricle and into the inferior vena cava (IVC) under fluoroscopy. 


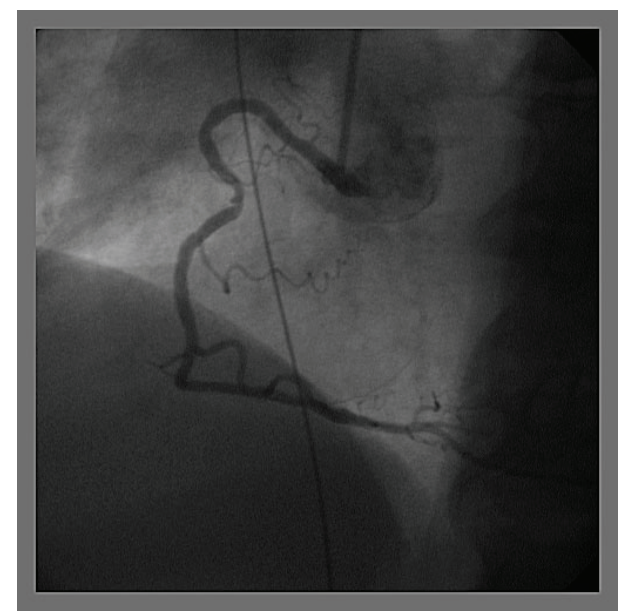

Figure 2. J-wire was seen spanning from the superior vena cava (SVC) to inferior vena cava (IVC) without looping in the right ventricle in 2007.

to IVC without looping in the right ventricle (Fig. 2). It was believed that the wire was left over from a right heart catheterization done at an outside institution at least seven years prior to his presentation. At that time, attempts to retrieve the wire using different percutaneous retrieval devices were unsuccessful. It was felt that the wire was endothelialized and/or layered with thrombus, so no further intervention was undertaken at that time. Given these presenting symptoms and migration of the J-wire cephelad, a repeat endovascular approach was attempted.

A venogram showed that the distal end of the wire was located $2 \mathrm{~cm}$ outside the IVC (Fig. 3). The J-wire then tracked up the IVC, where it appeared endothelialized until free floating into the right atrium and right ventricle. After looping inside the right ventricle it extended back into the right atrium up to the SVC. Percutanous approach was attempted with multiple different snaring devices and through

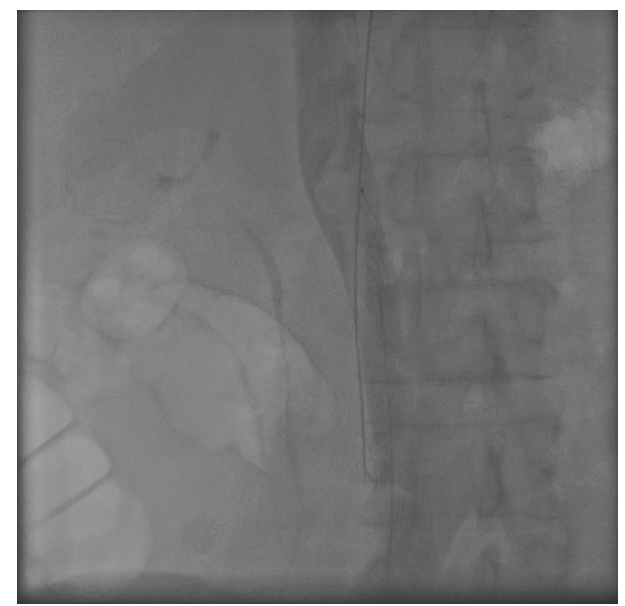

Figure 3. A venogram showed the distal end of the wire outside the inferior vena cava (IVC).

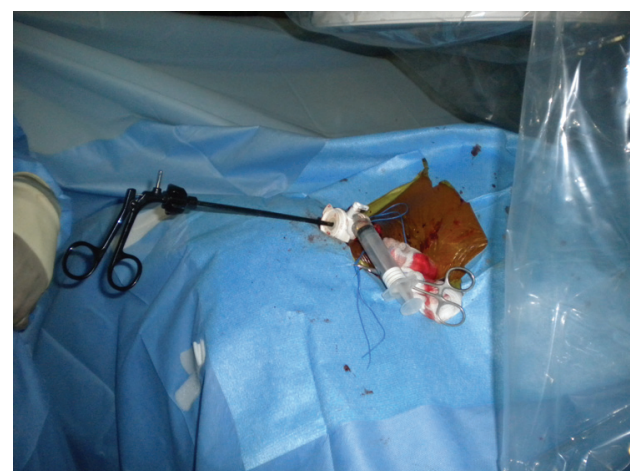

Figure 4. An Autosuture Versa Step $5 \mathrm{~mm}$ port was inserted through the 10 French sheath.

both left and right femoral vein access with no success.

Recurrent bouts of non-sustained ventricular tachycardia and the J-wire were believed to be the substrate for the arrhythmia. Removal of the wire was deemed necessary.

Vascular surgery was consulted and a right internal jugular approach through a 10 French sheath with different snaring devices was attempted intraoperatively with also no success. An Autosuture Versa Step 5mm port was inserted through the 10 French sheath and then a flexible alligator cystoscope forcep (Fig. 4) was inserted through the port with successful recovery of the J-wire (Fig. 5). The patient tolerated the procedure well and there were no further episodes of ventricular tachycardia. Chest x-ray showed no disruption of the Implantable Cardioverter-defibrillator (ICD) leads post wire removal.

\section{Discussion}

Over the last few decades, there has been an increase in the number of percutaneous cardiovascular interventions. Un-

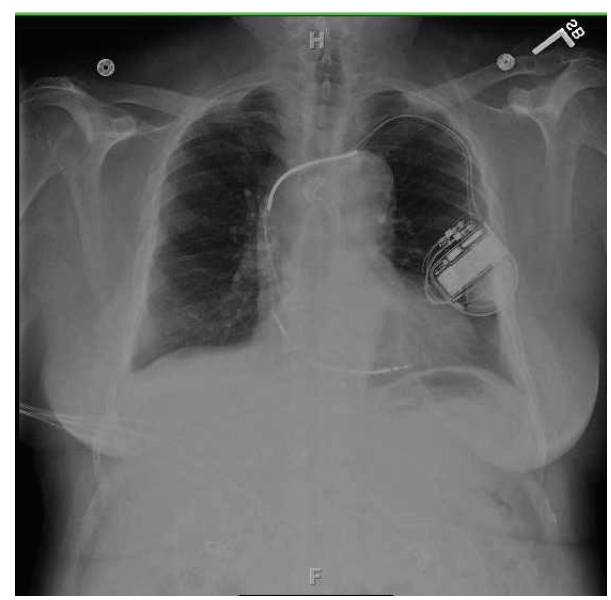

Figure 5. A flexible alligator cystoscope forcep was inserted through the port with successful recovery of the J-wire. 
fortunately, with this rise has come an increase in the incidence of lost or embolized foreign bodies in the central and peripheral circulation. The true incidence of retained foreign bodies is unknown, however it is estimated at $0.1 \%$ to $1.5 \%$ $[1,2]$. Examples of retained objects include J-wire, stents, Swan-Ganz catheters and pacemaker leads. Most common sites of retrieval are great veins, right heart and coronary circulation.

Although patients with the retained object may remain asymptomatic for many years, serious complications including arrhythmias, myocardial perforation, infection and thrombosis were well-reported [3]. Once symptomatic, removal of the object becomes necessary. The advances in the techniques of percutaneous intervention have made the retrieval of retained foreign bodies feasible, safe and effective, as well as limited the need for the need for surgery [4]. Devices used in percutaneous extraction of retained foreign bodies include loop-snares, retrieval baskets and grasping forceps. Once standard percutaneous tools fail, alternative techniques may prove to be of great use. Laparoscopic devices may sometimes be utilized to facilitate the removal process [5]. Trans-femoral approach is commonly used with the trans-jugular approach as a second line option if the former approach fails [5].

In our case, the patient had the J-wire migrate cephelad over two years until looped within the right ventricle and caused ventricular tachycardia. Because the distal end of the wire was endothelialized into the IVC, the extraction of the J-wire was unsuccessful using the trans-femoral and transjugular approach using conventional snares. The absence of a free wire tip available for snaring is the most common cause of failure [4]. The presence of ICD leads further complicated the procedure. Trans-jugular approach provided a port to the proximal free end of the wire. The fond knowl- edge of available tools and creative ideas to achieve a good patient outcome is paramount when dealing with endovascular procedural complication. To our knowledge, this is the first reported case of a novel utilization of laparoscopic tools for retrieval of a retained exchange wire via the trans-jugular approach.

Conflict of interest: No disclosures for any authors.

\section{References}

1. Bonvini RF, Rastan A, Sixt S, Noory E, Beschorner U, Leppanen O, Mach F, et al. Percutaneous retrieval of intravascular and intracardiac foreign bodies with a dedicated three-dimensional snare: a 3-year single center experience. Catheter Cardiovasc Interv. 2009;74(6):939945.

2. Burri C, Henkemeyer H, Passler HH. [Catheter embolism]. Schweiz Med Wochenschr. 1971;101(44):15751577 contd.

3. Fisher RG, Ferreyro R. Evaluation of current techniques for nonsurgical removal of intravascular iatrogenic foreign bodies. AJR Am J Roentgenol. 1978;130(3):541548.

4. Bessoud B, de Baere T, Kuoch V, Desruennes E, Cosset MF, Lassau N, Roche A. Experience at a single institution with endovascular treatment of mechanical complications caused by implanted central venous access devices in pediatric and adult patients. AJR Am J Roentgenol. 2003;180(2):527-532.

5. Mazzetti H, Cichero CF, Tentori MC, Mascheroni O. Transjugular approach for lead extraction. Europace. 2008;10(2):156-160. 